\title{
Avaliação da qualidade microbiológica de polpas de frutas congeladas do município
}

\section{de Limoeiro do Norte - Ceará}

Evaluation of the microbiological quality of frozen fruit pulps in the municipality of Limoeiro do

Norte - Ceará

Evaluación de la calidad microbiológica de pulpas de frutas congeladas en el municipio de Limoeiro

do Norte - Ceará

Recebido: 02/11/2021 | Revisado: 09/11/2021 | Aceito: 21/11/2021 | Publicado: 23/11/2021

Sebastiana Cristina Nunes Reges

ORCID: https://orcid.org/0000-0002-8720-1862 Instituto Federal de Educação, Ciência e Tecnologia do Ceará, Brasil E-mail: cristinanunesreges@gmail.com

Renata Chastinet Braga

ORCID: https://orcid.org/0000-0001-6630-2835 Instituto Federal de Educação, Ciência e Tecnologia do Ceará, Brasil

E-mail: rchastinet@gmail.com

Pahlevi Augusto de Souza

ORCID: https://orcid.org/0000-0002-7964-3193 Instituto Federal de Educação, Ciência e Tecnologia do Rio Grande do Norte, Brasil

E-mail: pahlevi10@hotmail.com

Germana Conrado de Souza

ORCID: https://orcid.org/0000-0002-5297-6465

Instituto Federal de Educação, Ciência e Tecnologia do Ceará, Brasil

E-mail: germanaconrado@ifce.edu.br

Elisabeth Mariano Batista

ORCID: https://orcid.org/0000-0001-5250-4110

Universidade Federal do Ceará, Brasil

E-mail: elisabethmariano@hotmail.com

Daiane dos Santos Pinto

ORCID: https://orcid.org/0000-0003-1475-4252

Universidade Federal do Ceará, Brasil

E-mail: daianesp2013@gmail.com

Zulene Lima de Oliveira

ORCID: https://orcid.org/0000-0001-5524-5644

Instituto Federal de Educação, Ciência e Tecnologia do Ceará, Brasil

E-mail: zuleneoliveira2907@gmail.com

Auriana de Assis Regis

ORCID: https://orcid.org/0000-0001-8901-0640

Universidade Estadual de Ponta Grossa, Brasil

E-mail: assisauriana@hotmail.com

\section{Resumo}

O mercado de polpa de frutas congeladas tem apresentado crescimento nos últimos anos, e para que esse mercado continue em expansão é fundamental que o produto possua qualidade satisfatória, principalmente do ponto de vista microbiológico. Por ser um produto alimentício com elevado percentual de água em sua composição, apresenta alta perecibilidade além de uma quantidade considerável de desperdício que podem desfavorecer economicamente o setor agroindustrial de polpas de frutas. Dessa maneira, o objetivo deste trabalho foi avaliar a qualidade microbiológica de polpas de frutas congeladas produzidas e comercializadas no município de Limoeiro do Norte - CE. Para isso, 100 amostras de polpas de frutas congeladas adquiridas no comércio e em uma empresa produtora, de 11 diferentes sabores, foram submetidas a análise microbiológica de coliformes totais, termotolerantes, contagem de bolores e leveduras e Salmonella sp. Todas as amostras apresentaram conformidade com a legislação para coliformes totais e termotolerantes, enquanto que $60 \%$ das amostras apresentaram contagem de bolores e leveduras acima do permitido $\left(7,9 \times 10^{5} \mathrm{UFC} / \mathrm{g}\right)$. Detectou-se a presença de Salmonella sp. em uma amostra de polpa sabor caju, obtida em estabelecimento comercial, estando em desacordo com a legislação vigente, que determina que este microrganismo deve estar ausente. Portanto, concluiu-se que em decorrência da qualidade microbiológica inadequada, a empresa produtora demonstrou controle higiênico-sanitário, seleção de matérias-primas e condições de armazenamento insatisfatórias no processamento das polpas de frutas congeladas produzidas e comercializadas em Limoeiro do Norte - CE, e portanto, oferecendo riscos à saúde dos consumidores.

Palavras-chave: Análise; Deterioração; Higiene; Vegetais. 


\begin{abstract}
The frozen fruit pulp market has been growing in recent years, and for this market to continue expanding, it is essential that the product has satisfactory quality, especially from a microbiological point of view. As it is a food product with a high percentage of water in its composition, it has high perishability and a considerable amount of waste that can economically disadvantage the agro-industrial fruit pulp sector. Thus, the objective of this work was to evaluate the microbiological quality of frozen fruit pulps produced and marketed in the municipality of Limoeiro do Norte - EC. For this, 100 samples of frozen fruit pulps acquired in the commerce and in a producing company, of 11 different flavors, were submitted to microbiological analysis of totals coliforms and thermotolerant, mold and yeast count and Salmonella sp. All samples were in compliance with the legislation for total and thermotolerant coliforms, while $60 \%$ of the samples had mold and yeast counts above the permitted level $\left(7.9 \times 10^{5} \mathrm{CFU} / \mathrm{g}\right)$. Detectou it is the presence of Salmonella sp. u ma pulp sample cashew flavor, obtained in a commercial establishment, being incompliance with the legislation in force, which determines that this microorganism should be absent. Therefore, it is concluded that due to inadequate microbiological quality, the producing company demonstrated hygienic-sanitary control, selection of raw materials and unsatisfactory storage conditions in the processing of frozen fruit pulps produced and marketed in Limoeiro do Norte - CE, and therefore, offering health risks to consumers.
\end{abstract}

Keywords: Analysis; Deterioration; Hygiene; Vegetables.

\title{
Resumen
}

El mercado de pulpa de fruta congelada ha ido creciendo en los últimos años, y para que este mercado siga expandiéndose es fundamental que el producto tenga una calidad satisfactoria, especialmente desde el punto de vista microbiológico. Al ser un producto alimenticio con un alto porcentaje de agua en su composición, presenta una alta perecebilidad y una cantidad considerable de residuos que pueden perjudicar económicamente al sector agroindustrial de la pulpa de frutas. Así, el objetivo de este trabajo fue evaluar la calidad microbiológica de pulpas de frutas congeladas producidas y comercializadas en el municipio de Limoeiro do Norte - CE. Para ello, 100 muestras de pulpas de frutas congeladas adquiridas en el comercio y en una empresa productora, de 11 sabores diferentes, fueron sometidas a análisis microbiológico de coliformes totales, termotolerantes, recuento de mohos y levaduras y Salmonella sp. Todas las muestras cumplieron con la legislación para coliformes totales y termotolerantes, mientras que el $60 \%$ de las muestras tenían recuentos de moho y levadura por encima del nivel permitido $\left(7,9 \times 10^{5} \mathrm{UFC} / \mathrm{g}\right)$. Detectou es la presencia de Salmonella sp. u ma pulpa de sabor muestra de anacardo, obtenido en un establecimiento comercial, siendo en el cumplimiento de la legislación en vigor, lo que determina que este microorganismo debe estar ausente. Por lo tanto, se concluye que debido a una calidad microbiológica inadecuada la empresa productora demostró control higiénico-sanitario, selección de materias primas y condiciones de almacenamiento insatisfactorias en el procesamiento de pulpas de frutas congeladas producidas y comercializadas en Limoeiro do Norte - CE, y por ende, ofrecer riesgos para la salud de los consumidores.

Palabras clave: Análisis; Deterioro; Higiene; Vegetal.

\section{Introdução}

O Brasil é o terceiro maior produtor mudial de frutas, produzindo uma grande diversidade de frutas tropicais, subtropicais e temperadas (Silva \& Abud, 2017). Segundo a Associação Brasileira de Fruticultura (2014), as frutas frescas não processadas representam 53\% do total da produção brasileira de frutas, das quais 3\% são destinadas ao abastecimento do mercado internacional. Além disso, do total de frutas frescas 47\% são processadas pela indústria de alimentícia.

Por sua vez as frutas são alimentos altamente perecíveis, com perdas estimadas em cerca de 30-40\% nos estágios de pós-colheita, beneficiamento e distribuição (Spagnol et al., 2018). Nesse contexto o processamento da polpa congelada é em uma excelente opção de aproveitamento do excedente desses vegetais, constituindo uma importante atividade agroindustrial do setor de alimentos que agrega valor às frutas e minimiza as perdas (Clerici \& Carvalho-Silva, 2011; Jesus et al., 2018; NeriNuma et al., 2018).

Segundo a legislação brasileira, polpa de fruta é definida como sendo o produto não fermentado, não concentrado ou diluído, obtido pelo esmagamento de frutos polposos. As frutas devem ser preparadas através de processos tecnológicos adequados que assegurem uma boa qualidade das suas características físico-químicas, nutricionais e microbiológicas, conforme estabelecido na Instrução Normativa n 49 de 26 de setembro de 2018 (Brasil, 2018) do Ministério da Agricultura Pecuária e Abastecimento (MAPA). 
As principais vantagens da industrialização de polpa de frutas é permitir o consumo de frutas nativas de regiões específicas em todo o país. Além de possibilitar o seu armazenamento e processamento nos períodos de entressafra, garantindo sua oferta durante todo o ano. Também é vantajoso a comercialização de polpas de frutas congeladas, visto que as mesmas são um produto de fácil preparo e uma fonte de compostos bioativos, vitaminas, minerais, fibras, entre outros constituintes benéficos para a saúde do consumidor (Spada et al., 2008, Castro et al., 2015).

Devido à sua composição, as polpas de frutas compõem-se em um substrato ideal para o desenvolvimento de microrganismo s alteradores e patogênicos que podem oferecer riscos para a saúde do consumidor. A contaminação microbiológica por microrganismos patogênicos ocorre durante o processamento, nas etapas de pré e pós-colheita (Alegbeleye et al., 2018). A contaminação de polpa de frutas está relacionada às falhas de higiene do manipulador e de equipamentos, utensílios e veículos de transporte. Além disso, o uso da água de lavagem contaminada, irregularidades no armazenamento e embalagens inapropriadas estão envolvidos na contaminação (Sant'anna et al., 2020).

Assim é de fundamental importância a avaliação dos parâmetros microbiológicos em polpas de frutas, para assegurar a qualidade do produto, visando atender não somente aos padrões estabelecidos pela legislação vigente, mas também às exigências do mercado consumidor. Diante do exposto, este trabalho teve como objetivo avaliar a qualidade microbiológica de polpas de frutas congeladas em estabelecimentos produtores e comercializadores do município de Limoeiro do Norte - CE.

\section{Metodologia}

\subsection{Coleta e preparo das amostras}

Foram coletadas amostras em estabelecimentos comerciais e em empresas produtoras de polpa de frutas no município de Limoeiro do Norte, Ceará, Brasil. As amostras foram coletadas em unidades de 500g, divididas em embalagens de $100 \mathrm{~g}$, a partir da embalagem original de comercialização, acondicionadas em recipientes de isopor com gelo e encaminhadas ao laboratório de microbiologia de alimentos do IFCE Campus Limoeiro do Norte- CE para realização das análises. No laboratório as amostras foram descongeladas em Béckers de 500mL e codificadas. Foram analisadas 100 amostras de polpas de fruta congeladas, sendo: 10 de maracujá, 10 de goiaba, 10 acerola, 10 de abacaxi, 10 de cajá, 5 de cajá-umbu, 10 de graviola, 10 de manga, 10 de caju, 10 de tamarindo e 5 de ameixa.

\subsection{Análise microbiológica}

As análises microbiológicas foram realizadas no Laboratório de Microbiologia de Alimentos do Instituto Federal do Ceará campus Limoeiro do Norte-CE. A caracterização dos microrganismos a serem determinados nas análises microbiológicas foi baseada nos padrões preconizados pela legislação vigente RDC n $^{\text { }} 12$ de 2 de janeiro de 2001 (Brasil, 2001). Foram realizadas as análises de contagem de bolores e leveduras, coliformes totais, coliformes termotolerantes e Salmonella sp. O tratamento estatístico dos resultados foi realizado com o auxílio do programa STATISTICA versão 7.1. Os resultados foram verificados através da análise de variância (ANOVA) e comparação múltipla das médias pelo teste de Tukey $(\mathrm{p}<0,05)$.

\subsubsection{Contagem de bolores e leveduras}

A contagem de bolores e leveduras foi realizada empregando a técnica de plaqueamento direto em superfície na qual as amostras de polpas de frutas congeladas foram diluídas e transferidos $10 \mathrm{~mL}$ para um frasco de $90 \mathrm{~mL}$ contendo solução salina (85\%) esterilizada, e em seguida após homogeneização, transferidas foram depositadas alíquotas de $1 \mathrm{~mL}$ com auxílio de pipetas. Posteriormente essas alíquotas foram espalhadas utilizando alça de Drigalsky, na superfíce do meio de cultura Ágar Batata Dextrose (BDA) acidificado a 10\%, seguido por incubação a $28{ }^{\circ} \mathrm{C} / 3-5$ dias. Após o período de incubação as colônias 
que apresentaram crescimento conforme exposto na Figura 1 foram submetidas à contagem com auxílio de uma lupa e os resultados foram registrados como unidades formadoras de colônias (APHA, 2013).

Figura 1- Contagem de bolores e leveduras em polpas de frutas.

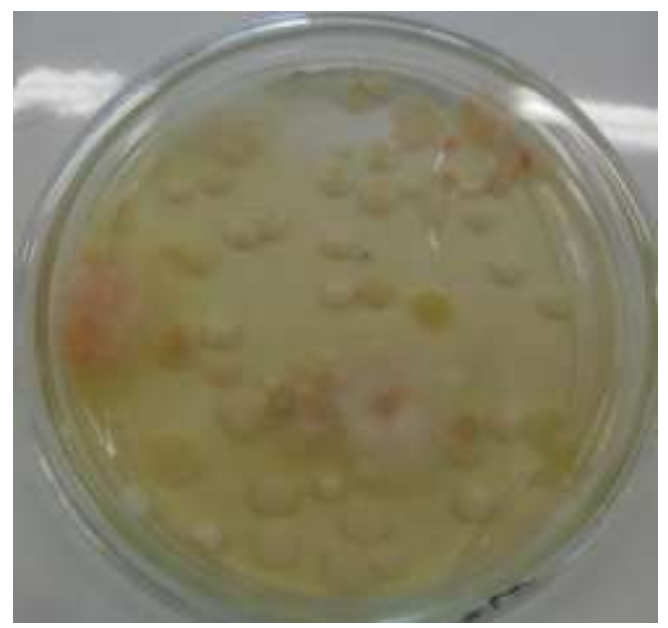

Fonte: Autores (2013).

\subsubsection{Coliformes totais e termotolerantes}

Para a quantificação de coliformes totais e termotolerantes, utilizou-se a técnica do Número Mais Provável (NMP) (APHA, 2013). Primeiramente foi realizado o teste presuntivo para coliformes totais, onde transferiu-se $25 \mathrm{~g}$ de polpa de fruta para erlenmeyer contendo $225 \mathrm{~mL}$ de água peptonada $0,1 \%$. A partir disso, transferiu-se $1 \mathrm{~mL}$ para tubos contendo $10 \mathrm{~mL}$ de Caldo Lauril Sulfato Triptose (LST) com tubos de Durhan invertidos. Em seguida eles foram incubados a $35^{\circ} \mathrm{C}-37^{\circ} \mathrm{C} / 24 \mathrm{~h}$. Após esse período, os tubos que apresentaram formação de gás, indicativo de coliformes totais, tiveram alíquotas transferidas tubos contendo $10 \mathrm{~mL}$ de Caldo Bile Verde Brilhante (BVB) e incubados a $35^{\circ} \mathrm{C}-37^{\circ} \mathrm{C} / 48 \mathrm{~h}$, para teste confirmativo de coliformes totais. Para verificar a presença de coliformes termotolerantes, transferiu-se uma alçada com o inóculo dos tubos de Caldo BVB com formação de gás, para tubos contendo caldo Escherichia coli (EC), seguido de incubação a $45^{\circ} \mathrm{C}$ por $24-48$ h em banho-maria. Quando verificado a produção de gás, o resultado foi considerado positivo para a presença de coliformes termotolerantes. A partir dos tubos positivos de caldo EC, foram inoculadas alíquotas em placas de petri contendo meio de cultura ágar eosina azul de metilo (EMB) e posteriormente incubadas a $35-37{ }^{\circ} \mathrm{C} / 24 \mathrm{~h}$. Por fim, a caracterização dos coliformes termotolerantes foi evidenciada pelo crescimento de colônias com centros enegrecidos e brilho verde metálico característico de E. coli em cultura de EMB.

\subsubsection{Salmonella sp.}

Para detecção de Salmonella sp., foram pesados $25 \mathrm{~mL}$ de polpa e transferidos para erlenmeyer contendo $225 \mathrm{~mL}$ de água peptonada, seguido de incubação a $35^{\circ} \mathrm{C} / 24 \mathrm{~h}$. Após o pré-enriquecimento transferiu-se 1 ml do cultivo para caldo Tetrationato (TT) e $0,1 \mathrm{~mL}$ para caldo Rappaport Vassiliadis (RV), sendo incubados a $35^{\circ} \mathrm{C} / 24 \mathrm{~h}$. Após o período de incubação, iniciou-se o plaqueamento diferencial, onde foi transferida uma alçada dos caldos TT e RV, para placas contendo Ágar Entérico Hectoen (HE), Ágar Bismuto Sulfito (BS), Ágar Xilose Lisina Desoxiciolato (XLD), Ágar Salmonella-Shigella (SS), Ágar Verde Brilhante (VB) e incubadas as placas invertidas em estufa de crescimento a 35 ' C/24 h. As colônias suspeitas de Salmonella sp. são identificadas através da mudança de coloração do meio de cultura (vermelho, laranja, púrpura e amarelo), que é resultante da reação bioquímica de determinados compostos químicos do meio que podem ou não serem assimilados pela bactéria através de seu metabolismo fermentativo. Seguindo o princípio de assimilação via metabolismo do 
microrganismo em assimilar compostos químicos dos meios de cultura, estas colônias foram transferidas para tubos contendo Ágar Tríplice Açúcar Ferro (TSI) e Ágar Lisina Ferro (LIA), seguida de incubação a $35^{\circ} \mathrm{C} / 24$ h. Os tubos com reações típicas que incluem alteração de cor (amarelo e púrpura) e produção de gás no tubo, foram submetidos a provas sorológicas e bioquímicos (Apha, 2013).

\section{Resultados e Discussão}

\subsection{Coliformes totais e termotolerantes}

Os resultados da análise de coliformes totais e termotolerantes em polpas de frutas coletadas em empresas produtoras e no comércio do município de Limoeiro do Norte - CE são apresentados na Tabela 1.

Tabela 1 - Resultados da análise de coliformes totais e termotolerantes em polpas de frutas coletadas na empresa produtora e comércio do município de Limoeiro do Norte - CE. *NMP/g - Número Mais Provável por grama.

\begin{tabular}{|c|c|c|c|c|}
\hline Polpa & $\begin{array}{c}\text { Coliformes totais } \\
\text { (NMP/g) }\end{array}$ & $\begin{array}{c}\text { Coliformes } \\
\text { termotolerantes } \\
(\mathrm{NMP} / \mathrm{g})\end{array}$ & $\begin{array}{r}\text { Coliformes } \\
\text { totais }(\mathrm{NMP} / \mathrm{g})\end{array}$ & $\begin{array}{c}\text { Coliformes } \\
\text { termotolerantes } \\
\text { (NMP /g) }\end{array}$ \\
\hline & \multicolumn{2}{|c|}{ Empresa produtor } & \multicolumn{2}{|c|}{ Comércio } \\
\hline Maracujá & $<3$ & $<3$ & $<3$ & $<3$ \\
\hline Goiaba & $<3$ & $<3$ & $<3$ & $<3$ \\
\hline Acerola & $<3$ & $<3$ & $<3$ & $<3$ \\
\hline Abacaxi & $<3$ & $<3$ & $<3$ & $<3$ \\
\hline Cajá & $<3$ & $<3$ & $<3$ & $<3$ \\
\hline Cajá-umbu & $<3$ & $<3$ & - & - \\
\hline Graviola & $<3$ & $<3$ & $<3$ & $<3$ \\
\hline Manga & $<3$ & $<3$ & $<3$ & $<3$ \\
\hline Caju & 9 & $<3$ & 4 & 4 \\
\hline Tamarindo & $<3$ & $<3$ & $<3$ & $<3$ \\
\hline Ameixa & - & - & $<3$ & $<3$ \\
\hline
\end{tabular}

Fonte: Autores (2013).

A resolução RDC $n^{\circ} 12$ de janeiro de 2001, que dispõe sobre o padrão microbiológico para alimentos no Brasil, estabelece como máximo tolerável de coliformes termotolerantes, em amostra indicativa, de polpas de frutas $10^{2} \mathrm{NMP} / \mathrm{g}$, conforme o item 1c do Anexo 1 dessa resolução. Os resultados das análises da presente pesquisa (Tabela 1) demonstraram que nas contagens de coliformes totais, observou-se que as polpas se apresentaram dentro dos padrões determinados pela legislação, sendo que os maiores índices foram obtidos na empresa produtora (9 NMP/g). Quanto aos resultados para coliformes termotolerantes no comércio, verificou-se que $12 \%$ apresentaram resultado positivo, sendo: 2 polpas de acerola, 3 de caju e 1 de manga. Dentre elas, 3 (1 de manga e 2 de caju) confirmaram a presença de coliformes termotolerantes verificada através da produção de gás no interior dos tubos de Duhram contidos em tubos de ensaio com meio de cultura caldo EC. Obteve-se valores para esta contagem de $4 \mathrm{NMP} / \mathrm{g}$, estando dentro dos padrões microbiológicos preconizados pela legislação. 
Silva et al. (2020) analisando as características microbiológicas de 30 amostras de polpas de frutas congeladas comercializadas em supermercado de Belém- PA, quanto aos microrganismos coliformes termotolerantes e Salmonella spp., observaram que 93,34\% das amostras encontravam-se em conformidade em relação a legislação vigente para coliformes termotolerantes, além de ausência de Salmonella spp. em 25 g, em 100\% das amostras analisadas. Relacionando este resultado com aqueles verificados por Alvarenga et al. (2017) analisando a qualidade microbiológica de 60 amostras de polpas de frutas congeladas de 6 diferentes sabores, industrializadas no município de Muriaé -MG, não observaram contagem para coliformes termotolerantes, detectando a presença em 50\% das amostras (polpas de abacaxi, morango e caju) resultado positivo para coliformes totais em decorrência de possíveis falhas no processamento e armazenamento desses produtos. Em relação a presente pesquisa a polpa de caju demonstrou resultado semelhante ao verificado pelos autores. Os resultados obtidos por Santos et al. (2017) assemelham-se aos verificados nesta pesquisa que ao analisar a qualidade microbiológica de três marcas (A, B e C) de polpas de frutas congeladas (acerola, manga e maracujá), em Vitória da Conquista- BA, utilizando a técnica de filme Petri, observaram que as polpas de frutas analisadas encontravam-se dentro dos padrões estabelecidos pela legislação vigente com relação a coliformes totais e E. coli, e Salmonella sp.

Conforme Silva et al. (2020) o índice referente a contagem de coliformes termotolerantes é utilizado para avaliar as condições higiênicas de toda cadeia produtiva que envolve desde a produção até o processamento e estocagem, sendo necessário seguir diretrizes de boas práticas de produção e comercialização de produtos. A presença deste grupo de microrganismo sugere contaminação por Escherichia coli, que possui como habitat o intestino humano e de outros animais endotérmicos.

\subsection{Salmonella sp.}

Os resultados da pesquisa de Salmonella sp. realizada em amostras de polpas de frutas coletadas diretamente da empresa produtora e em estabelecimentos comerciais do município de Limoeiro do Norte, são apresentados na Tabela 2.

Tabela 2 - Resultado da pesquisa de Salmonella sp. em amostras de polpas de frutas coletadas da empresa produtora e comércio do município de Limoeiro do Norte - CE.

\section{Pesquisa de Salmonella sp.}

\section{Polpa}

\begin{tabular}{lcc} 
& Empresa produtora & Comércio \\
\cline { 2 - 3 } Maracujá & Ausente & Ausente \\
Goiaba & Ausente & Ausente \\
Acerola & Ausente & Ausente \\
Abacaxi & Ausente & Ausente \\
Cajá & Ausente & Ausente \\
Cajá-umbu & Ausente & Ausente \\
Graviola & Ausente & Ausente \\
Manga & Ausente & Ausente \\
Caju & Ausente & Presente \\
Tamarindo & Ausente & Ausente \\
Ameixa & Ausente & Ausente \\
\hline
\end{tabular}


Para os resultados obtidos nesta presente pesquisa observou-se a ausência de Salmonella sp. para as amostras de polpas de frutas congeladas da empresa produtora, mostraram que este microrganismo apresentou resultado negativo em todas as amostras avaliadas. Diante dos resultados pode-se expor que a empresa tem adotado condições higiênico-sanitárias durante o processamento de polpas de frutas, operações de limpeza, escolha das matérias-primas e condições de armazenamento, estando em conformidade com as Boas Práticas de Fabricação (BPF). Contudo, as polpas coletadas nos estabelecimentos comerciais, observou-se que este microrganismo foi detectado em uma amostra de polpa sabor caju. A bactéria Salmonella sp. por ser um microrganismo patogênico, deve estar ausente em $25 \mathrm{~mL}$ da amostra (Brasil, 2001) sendo sua presença em alimentos, um indicativo de armazenamento em condições higiênico-sanitárias insatisfatórias, caracterizandose em risco à saúde do consumidor. As polpas coletadas nas empresas produtoras, este microrganismo manifestou-se ausente em 100\% das amostras analisadas. Dessa forma, evidencia-se a necessidade da efetiva fiscalização pela Vigilância Sanitária, bem como de suporte técnico profissional e treinamento dos manipuladores, a fim de assegurar um produto com qualidade e segurança para o consumidor.

\subsection{Contagem de bolores e leveduras}

Os resultados médios da contagem de bolores e leveduras das polpas congeladas de frutas estão expostos na Tabela 3 . Observa-se que das 100 amostras analisadas todas apresentaram contaminação por bolores e leveduras, com valores variando de $10^{2}$ até $7,9 \times 10^{5} \mathrm{UFC} / \mathrm{g}$. Esta ocorrência pode ser atribuída ao elevado teor de carboidratos normalmente presentes nas polpas de frutas, além do caráter ácido das polpas que propiciam o meio adequado para desenvolvimento desses microrganismos.

Tabela 3 - Valores médios da contagem de bolores e leveduras em amostras de polpas de frutas coletadas da empresa produtora e comércio do município de Limoeiro do Norte-CE.

\begin{tabular}{|c|c|c|}
\hline Polpa & \multicolumn{2}{|c|}{ Bolores e leveduras (UFC/g) } \\
\hline & Empresa Produtora & Comércio \\
\hline Maracujá & $1,5 \times 10^{5 *}$ & $3,3 \times 10^{4 *}$ \\
\hline Goiaba & $3,7 \times 10^{5 *}$ & $1,3 \times 10^{5 *}$ \\
\hline Acerola & $1,0 \times 10^{4 *}$ & $2,9 \times 10^{5 *}$ \\
\hline Abacaxi & $3,6 \times 10^{5 *}$ & $3,3 \times 10^{5 *}$ \\
\hline Cajá & $3,3 \times 10^{2}$ & $1,3 \times 10^{3}$ \\
\hline Cajá-umbu & $1,1 \times 10^{3}$ & - \\
\hline Graviola & $9,3 \times 10^{2}$ & $4,4 \times 10^{2}$ \\
\hline Manga & $1,5 \times 10^{4 *}$ & $1,6 \times 10^{4 *}$ \\
\hline Caju & $8,9 \times 10^{3 *}$ & $2,9 \times 10^{4 *}$ \\
\hline Tamarindo & $3,2 \times 10^{2}$ & $4,4 \times 10^{2}$ \\
\hline Ameixa & - & $2,4 \times 10^{2}$ \\
\hline
\end{tabular}

*Fora dos padrões estabelecidos segundo a Instrução Normativa n ${ }^{\circ} 01$ de 2000 (MAPA). Fonte: Autores (2013). 
Conforme as contagens de bolores e leveduras obtidas nas polpas analisadas, na Tabela 3, $60 \%$ das amostras, sendo os sabores: maracujá, goiaba, acerola, abacaxi, manga e caju, tanto na empresa quanto no comércio, não se enquadraram nos padrões estabelecidos pela Instrução Normativa $n^{\circ} 01$ de 2000 (Brasil, 2000) do Ministério da Agricultura Pecuária e Abastecimento - MAPA, na qual estabelece que a polpa de fruta deverá observar os limites máximos para a soma de bolores e leveduras de $5 \times 10^{3} / \mathrm{g}$ para polpa "in-natura", congelada ou não, e $2 \times 10^{3}$ para polpa conservada quimicamente e/ou que sofreu tratamento térmico, por apresentarem resultados acima do padrão microbiológico exigido. Comparando os resultados obtidos nesta pesquisa com aqueles verificados por Santos \& Vieira (2020) observou-se resultado semelhante em polpas de frutas artesanais produzidas e comercializadas nos mercados públicos do município de João Pessoa- PB, apresentando percentual de contagem de $87,5 \%$ das amostras que encontraram-se acima do valor estabelecido na legislação. Percentual menos elevado foi verificado por Faria et al. (2016) que avaliando a qualidade microbiológica de cinco marcas (A, B, C, D, E) de polpas de frutas congeladas (abacaxi, acerola, caju, goiaba e maracujá), comercializadas na cidade de Cuiabá - MT para comparar aos padrões da legislação vigente observaram que a contagem de bolores e leveduras, três amostras (12\%) apresentaram índices elevados, acima do limite máximo estabelecido pela legislação.

Resultados semelhante à presente pesquisa foi observado por Santos et al. (2017) observaram crescimento significativo de

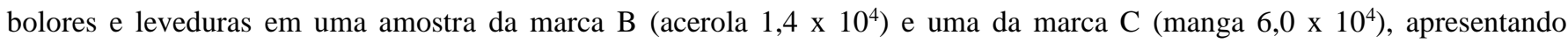
resultados acima dos valores permitidos pela legislação, o que sugere a necessidade urgente de adequação do processo produtivo. Conforme os autores, a presença de bolores e leveduras em $22 \%$ das amostras, remete à necessidade de um controle mais rigoroso no processo de fabricação e acaba refletindo na qualidade do produto final, podendo colocar em risco a saúde do consumidor. Porém resultado satisfatório da qualidade de polpas de frutas congeladas em Salgueiro -PE, foi verificado por Araújo, Alves \& Marques (2018) que observaram conformidade com os padrões da legislação em 100\% das amostras para estes microorganismos. A polpa de acerola da marca A foi a única que apresentou ausência de bolores e leveduras.

Moraes \& Machado (2021) em uma revisão panorâmica de estudos publicados com análises microbiológicas de polpas de frutas congeladas realizada no período de 2010-2020, observaram que dentre os parâmetros microbiológicos mais avaliados foram os bolores e leveduras com $67,7 \%$ das pesquisas realizadas no Brasil, em dez anos. Segundo os autores pelo menos uma amostra encontrava-se em desacordo com a legislação, destacando a necessidade do controle do processamento de polpas de frutas através da inspeção e vigilância no país.

\section{Conclusão}

As polpas de frutas obtidas tanto na empresa produtora quanto no comércio, encontram-se dentro dos padrões estabelecidos pela legislação vigente com relação a coliformes totais e termotolerantes. Porém, 60\% das amostras apresentaram valores acima do permitido para bolores e leveduras. Dentre as polpas comercializadas uma delas (sabor caju) apresentou contaminação por Salmonella sp., provavelmente em decorrência de falhas higiênicas durante a manipulação e armazenamento do produto.

O objetivo desta pesquisa foi alcançado tendo em vista que por meio desta, foi possível concluir que a qualidade das polpas de frutas congeladas, em estabelecimentos produtores e comercializadores destes produtos, necessita ser controlada e mantida por meio da adoção de boas práticas de fabricação afim de evitar que a contaminação microbiana detectada comprometa esses produtos e reduza os riscos para a saúde dos consumidores.

Ressalta-se a importância de serem realizados trabalhos futuros acerca da avaliação da qualidade microbiológica das

polpas de frutas, tanto as comercializadas como nas empresas produtoras e/ou beneficiadoras. Evidencia-se ainda, a necessidade da adoção e aplicação de ferramentas da segurança alimentar, como a adoção do manual de Boas Práticas de 
Fabricação (BPF), a fim de assegurar a adequação do processamento às exigências higiênico-sanitárias preconizadas em lei visando dessa forma, a obtenção de produtos de qualidade e sanidade para os consumidores.

\section{Referências}

Alvarenga, M. V., Vieira, L. J., Santos, R. J. A. \& Fernandes, F. M (2017). Análise microbiológica de polpas de frutas congeladas e industrializadas. Revista Científica da Faminas. RC Faninas. 12(3), 1-6.

Alegbeleye O. O., Singleton, I. \& Sant'ana, A. S. (2018). Sources and contamination routes of microbial pathogens to fresh produce during field cultivation: a review. Food Microbiology, 73, 177-208.

APHA - American Public Health Association. Compendium of Methods for Microbiological Examination of Foods. 6 ed. Washington, DC: American Public Health Association, 2013.

Araújo, E. C. O., Alves, J. E. A. \& Marques, L. C (2018). Avaliação de parâmetros de qualidade de polpas de frutas congeladas comercializadas no município de Salgueiro- PE. Revista Semiárido de Visu. Petrolina, 6(1), 4-11.

Associação Brasileira de Fruticultura. Anuário Brasileiro da Fruticultura 2014. Santa Cruz do Sul: Editora Gazeta.

Brasil. Ministério da Agricultura, Pecuária e Abastecimento (2018, setembro 26). Resolve: Fica estabelecida em todo o território nacional a complementação dos Padrões de Identidade e Qualidade de Suco e Polpa de Fruta, na forma desta Instrução Normativa (Instrução Normativa ${ }^{\circ}$ 49). Diário Oficial da União, Brasília.

Brasil. Agência Nacional de Vigilância Sanitária. Resolução RDC no 12, de 02/01/2001. Regulamento Técnico sobre padrões microbiológicos para alimentos. Diário Oficial da República Federativa do Brasil, Brasília, DF, 10 janeiro de 2001.

Castro, T. M. N., Zamboni, P. V., Dovadoni, S., Cunha Neto, A. \& Rodrigues, L. J. (2015). Parâmetros de qualidade de polpas de frutas congeladas. Revista do Instituto Adolfo Lutz, 74(4), 426-436.

Clerici, M. T. P. S. \& Carvalho-Silva, L. B. (2011). Nutritional bioactive compounds and technological aspects of minor fruits grown in Brazil. Food Research International, 44, 1658-1670.

Faria, R. A. P. G.; Brasil, A. S.; Rosa, O. O. \& Siqueira, N. M. F. P (2016). Avaliação da qualidade microbiológica de polpas de frutas congeladas comercializadas na cidade de Cuiabá- MT. Revista Higiene Alimentar, 30(256/257), 128-131.

Jesus, A. L. T., Leite, T. S., Cristianini, M. (2018). High isostatic pressure and thermal processing of açaí fruit (Euterpe oleracea Martius): effect on pulp color and inactivation of peroxidase and polyphenol oxidase. Food Research International, 105, 853-862.

Moraes, J. O. \& Machado, M. R. G. Qualidade microbiológica de polpa de fruta no Brasil: Um panorama dos anos 2010-2020. Research, Society and Development, v. 10, n. 7, e17110716377, 2021 (CC BY 4.0) | ISSN 2525-3409 | DOI: http://dx.doi.org/10.33448/rsd-v10i7.16377.

Neri-Numa, I. A., Sancho, R. A. S., Pereira, A. P. A. \& Pastore, G. M. (2018). Small Brazilian wild fruits: nutrients, bioactive compounds, health-promotion properties and commercial interest. Food Research International, 103, 345-360.

Spagnol, W. A., Silveira Junior, V., Pereira, E. \& Guimaraes Filho, N. (2018). Redução de perdas nas cadeias de frutas e hortaliças pela análise da vida útil dinâmica. Brazilian Journal of Food Technology, 21, e2016070.

Spada, P. D., Souza, G. G. N., Bortolini, G. V., Henriques, J. A. \& Salvador, M. (2008). Antioxidant, mutagenic, and antimutagenic activity of frozen fruits. Journal of Medicinal Food, 11(1), 144-151.

Santos, E. C. G.; Santana, R. F.; Viana Júnior, N. M. \& Miranda, A. S (2017). Determinação da qualidade microbiológica de polpa de fruta congelada utilizando a técnica de filme de Petri. Revista Brasileira de Produtos Agroindustriais. 19(3), 247-253.

Santos, R. E. \& Vieira, P. P. F (2020). Avaliação da qualidade microbiológica de polpa de fruta artesanal produzida e comercializadas nos mercados públicos de João Pessoa. Revista Brazilian Journal of Development, 6(9), 1-11.

Sant'anna, P. B., Franco, B. D.G., Maffei, D. F. (2020). Microbiological safety of ready-to-eat minimally processed vegetables in Brazil: an overview. Journal of the Science of Food and Agriculture, 100(13), 1-11.

Silva, C. E. D. F., \& Abud, A. K. D. S. (2017). Tropical fruit pulps: processing, product standardization and main control parameters for quality assurance. Brazilian Archives of Biology and Technology, 60, 1-19.

Silva, T. B. D.; Martins, V. S.; Pereira, J. M. C.; Brasil, L. S. N. \& Silva, S. M. R (2020). Análise microbiológica de polpas de frutas industrializadas comercializadas em supermercados de Belém -PA. Revista Univap, 26(50), 36-46. 